\title{
Insight-related beliefs and controllability appraisals contribute little to hallucinated voices: a transdiagnostic network analysis study
}

\author{
Elisavet Pappa ${ }^{1,4} \cdot$ Emmanuelle Peters $^{2,3} \cdot$ Vaughan Bell $^{3,4}$ (D)
}

Received: 6 February 2020 / Accepted: 7 July 2020 / Published online: 14 July 2020

(c) The Author(s) 2020

\begin{abstract}
Hallucinated voices cause high levels of distress and disability. Current theories suggest that insight-related beliefs, about internal or external origin, perceived source location, and appraisals of controllability are important in mediating the impact of these experiences but previous findings have been mixed. We report two open code and open data network analytic studies of items in the Psychotic Symptoms Ratings Scale for auditory verbal hallucinations (PSYRATS-AH) in a large sample of patients with hallucinated voices to examine the network structure of items at (1) first assessment, and (2) differences over two consecutive assessments during a wait-list period. Networks were generated using least absolute shrinkage and selection operator (LASSO) and extended Bayesian information criterion (EBIC) with node predictability. In Study $1(N=386)$, we report that insight-related items made a negligible contribution to hallucinated voices and the controllability appraisal made at most a modest contribution. Items relating to distress and negative content were the most central and most predicted by the wider network. In Study $2(N=204)$, we tested the longitudinal stability of the structure of hallucinated voices over a period of several months, finding a small change in total hallucination score and global strength but no clear evidence for an alteration in the structural relationship. The insight-related and controllability items remained as least influential over time. Insight-related beliefs and controllability appraisals may contribute less than previously thought to distressing hallucinated voices although we do not discount that other appraisals may remain important.
\end{abstract}

Keywords Psychosis $\cdot$ Network analysis $\cdot$ Predictability $\cdot$ Metacognition $\cdot$ Auditory hallucinations

\section{Background}

Electronic supplementary material The online version of this article (https://doi.org/10.1007/s00406-020-01166-3) contains supplementary material, which is available to authorized users.

Vaughan Bell

Vaughan.Bell@ucl.ac.uk

1 Department of Psychiatry, University College London, London, UK

2 Department of Psychology, Institute of Psychiatry, Psychology and Neuroscience, King's College London, London, UK

3 Psychological Interventions Clinic for Outpatients with Psychosis (PICuP), South London and Maudsley NHS Foundation Trust, London, UK

4 Research Department of Clinical, Educational and Health Psychology, UCL Centre for Clinical Psychology, University College London, Gower Street, London WC1E 6BT, UK
Auditory verbal hallucinations are present in up to $70 \%$ of patients with a diagnosis of schizophrenia, almost a quarter of patients with bipolar disorder, and contribute to both distress and disability in affected people [1]. However, the differing contributions of appraisals, perceptual and affective components are still poorly understood and a better understanding of the interrelationships between these components has been highlighted as a research priority [2].

The relationship between appraisals and other characteristics of hallucinated voices has been of interest due to their potential role in mediating impact. Traditionally, one of the most important appraisals relates to the perceived origin of voices, with 'insight' ascribed to the voice hearer if they believe the voices are generated within the self and 'lack of insight' ascribed to the voice hearer if they believe the voices originate from external sources [3]. The perceived auditory 'location' of the voices has also been considered to be a marker of clinical significance, with those perceived 
as coming from outside the head categorised as 'true hallucinations' and those perceived to be coming from inside the head designated as 'pseudohallucinations' [4]. Notably, some non-diagnostic cognitive models also stipulate internal versus external attributions as importing in determining whether the experience becomes a psychotic symptom [5]. The importance of location appraisals for judging hallucination severity has received mixed support. Stephane et al. [6] found that patients with schizophrenia who heard hallucinations inside their head were more likely to demonstrate memory source monitoring deficits, while Docherty et al. [7] found that internally located hallucinations were more intrusive and distressing. However, no reliable associations were found by Copolov et al. [4] in large sample of patients with psychotic disorders, neither were they found by Oulis et al.'s [8] study on patients admitted with acute psychosis, and they were only associated with number of hallucinated words and utterances in a study by Nayani and David [9]. Despite these mixed results, these characteristics are still cited in contemporary psychiatric textbooks as unequivocal markers of clinical severity $[10,11]$.

Morrison et al. [12] have cited metacognitive beliefs relating to the controllability of thoughts as a key component in hallucination proneness. A recent meta-analysis [13] examined judgements about the controllability of thoughts, the controllability of the person's own mind, as well as confidence in the accuracy of the person's own mind, and found only a moderate association between these factors and distress in clinical samples-which was reduced to a weak association when co-morbid symptoms were controlled for. However, appraisals regarding perceived controllability of voices themselves, rather than thoughts in general, have been found to characterise clinical as opposed to non-clinical voice hearers [14] (i.e. people not distressed or impaired by their voices), patients with first episode psychosis [1] and patients with more severe voice hearing symptoms [7].

Nevertheless, one drawback with existing studies in this area is that they have tended to rely on composite measures of severity that sum a range of characteristics like distress, intrusiveness and insight into a single metric-potentially obscuring structural relationships and interactions between insight-related beliefs, appraisals, and the affective and perceptual components of voices. Similarly, many studies are cross-sectional in nature meaning it is not possible to see how the relationship between different characteristics changes over time-something that requires longitudinal analysis.

One way of more effectively addressing the interaction of components in psychopathology is through the use of network analysis which estimates plausible candidates for causal interactions based on statistical relationships between symptom component measures, controlled for every other variable in the network [15]. One additional advantage of this method is that the potential importance of each element for the overall function and coherence of the network can be estimated using graph theoretic metrics [16]. Such networks therefore provide estimates of the significance of each characteristic in the overall network and potentially suggest beneficial points of intervention. However, to date, only a handful of studies have applied this method to understanding psychosis. For example, Isvoranu et al. [17] examined the inter-relationships between trauma and psychotic symptoms, Bell and O'Driscoll [18] and Murphy et al. [19] examined paranoia and the wider psychosis phenotype respectively in the general population, and van Rooijen et al. [20] examined psychosis-related symptoms in patients with psychosis. None to date have directly investigated hallucinated voices.

Consequently, we applied network analysis to examine the contribution of appraisals about origin, perceived source location, and controllability, to the structure and temporal stability of auditory hallucinations networks in a large sample of patients with psychosis using items from the Psychotic Symptom Rating Scales for Voices (PSYRATS-AH; [21]). We report two studies. For the first, we examined the relative importance of each of these components in a large sample of patients with hallucinated voices who attended an initial assessment at a clinic for psychological interventions for psychosis. For the second, following Santos et al. [22] we tested the longitudinal stability of the network in a subsample of patients who were followed-up over time while they were on a waiting list for intervention, allowing us to examine how these factors alter over time.

\section{Methods}

\section{Setting}

Data were collected from clinical assessments from 2003 to 2018 at the Psychological Interventions Clinic for outpatients with Psychosis (PICuP) based at the Maudsley Hospital in the South London and Maudsley National Health Service Foundation Trust. PICuP accepts referrals mainly from four boroughs in South London. Referral criteria for PICuP is "a diagnosis or suspected diagnosis of psychosis (including bipolar disorder), or presence of psychotic symptoms (for instance as a result of trauma)". Referrals are screened and those with confirmed psychotic symptoms are invited for an assessment. They are a highly diverse group in terms of social, economic, forensic, health and mental health (previously described in Peters et al. [23]).

\section{Sample and procedures}

For Study 1, we included patients who reported auditory hallucinations at the point of initial assessment (indicated 
by a positive PSYRATS-AH score) and had no missing data on any scale item, which left a total of 386 patients included in the analysis.

For the longitudinal analysis in Study 2, we included patients who had a positive PSYRATS-AH score at initial assessment and complete PSYRATS-AH assessments at both initial and second assessments, which left 204 patients included in the analysis. These two assessments are the initial assessments that form part of several standard clinical assessments that occur during the process of therapy. The first assessment occurs when patients are initially referred to the service and are added to the waiting list. The second, pre-therapy assessment occurs just prior to being allocated a therapist for the start of treatment. While on the waiting list the patient is living in the community, either under the care of a community mental health team or a general practitioner.

Assessments are conducted by assistant psychologists trained in conducting the evaluations that includes the PSYRATS-AH and several other measures with the full assessment lasting approximately 45-90 min. Demographic data are collected through the standard 'Patient Registration Form'. Ethical approval for the use of these data in research was granted by the London-Dulwich Research Ethics Committee (ref: 15/LO/1831) and only patients who consent to their anonymised data being used in research are included in the database. Therefore, the study has been conducted in accordance with the ethical standards laid down in the 1964 Declaration of Helsinki and its later amendments.

\section{Measures}

Auditory hallucinations were measured with the Psychotic Symptom Rating Scale for Voices (PSYRATS-AH; [21]) a widely used and clinically validated measure of auditory verbal hallucinations (internal consistency; $\alpha=0.75$ ) that takes the form of a semi-structured, 11-item, clinician-rated interview [24]. Each item is rated on a five-point ordinal scale from 0 (absent) to 4 (severe) with overall score ranging from 0-44 and includes items measuring frequency, disruption of life, duration, loudness, amount and degree of negative content (two items), amount and intensity of distress (two items), location (i.e., whether the voices sound like they are coming from inside or outside the head), belief about origin of the voices (i.e., whether they are internally or externally generated), and perceived controllability over the voices (i.e. can they bring them on or dismiss them). Each item on the PSYRATS-AH was included as a separate node in the network analysis.

\section{Statistical analysis and data availability}

The statistical programming language $R$ (version 3.5.3) was used to conduct all analyses. Open data and code have been made available that allow all main analyses to be reproduced. However, we have not included some demographic and descriptive clinical variables in the open data set to insure against re-identification. All analyses were completed on a 64-bit $\times 86$ Linux platform. The analysis code and nonidentifiable data for reproducing the analyses is available online at: https://osf.io/qkn6z/

For the first analysis, we used the mgm package (version 1.2 .5 ; [25]) to generate mixed graphical models to estimate the network using Least Absolute Shrinkage and Selection Operator (LASSO; [26]) using Extended Bayesian Information Criterion (EBIC) to select the best model [27]. This method produces an estimated network structure that maximises the probability of deriving the genuine structure in the population. In addition, we also calculated predictability for each node in each network [28, 29]. Predictability indicates how the value of each node is predicted by connected nodes potentially giving a more plausible metric of practical importance in terms of targets for interventions [29]. High predictability indicates that variance in a node is determined by mutual interactions between connected nodes. In summary, centrality metrics attempt to estimate how much the node affects the network, whereas predictability metrics attempt to estimate how much the network affects the node.

In the network visualisations, strengths of associations are represented by the thickness of the lines between nodes with positive relationships in green and negative relationships in red. The extent of node predictability is visualised by a ring (equivalent to a 'donut graph') around the edge of each node. Network layouts were generated using the qgraph (version 1.6 .1 ; [30]) using the Fruchterman-Reingold algorithm [31].

We subsequently estimated the following centrality metrics for each node [32] using qgraph: betweenness, referring to the number of times that a node lies in the shortest path between two other nodes in the network; closeness, defined as the average distance from a node to all other nodes in the network; strength, as the sum of weighted correlation coefficients of all the edges connected to a node; and expected influence, a measure of centrality that adjusts for the combined influence of positive and negative associations [33].

For Study 2, we compared total PSYRATS-AH scores between assessments using a paired t-test and Pearson's $r$. We then generated networks for the first and second assessments using the same statistical approach as described in Study 1. Following Santos et al. [22] these networks were subsequently compared using NetworkComparisonTest package for $R$ (NCT; [34]) modified for ordinal data [35]. The NCT is a two-tailed permutation test that uses random regrouping of participants from the networks and calculates the differences between the networks $[34,36]$. We tested for statistical difference in: (1) invariant network structure-whether the overall network structure is significantly different between time points; (2) edge strength, testing 
whether edges are different across networks; and (3) global strength - whether the overall level of connectivity is different. We also estimated centrality metrics and predictability values for each node in the two networks.

Finally, we assessed the stability of centrality metrics using an $m$ out of $n$ bootstrap method using the bootnet package [37] to estimate edge-weight reliability and a bootstrap analyses to estimate edge-weight reliability for $\mathrm{mgm}$ networks using the technique reported in Fried et al. [38].

\section{Results}

\section{Study 1: Network structure of auditory verbal hallucinations}

\section{Descriptive statistics}

Of the 386 included patients, 210 (55.4\%) were male, 169 $(44.6 \%)$ were female, with seven patients recorded as having missing data (which may include 'prefer not to say'). The mean age of patients was $40.84(\mathrm{SD}=10.2)$. Ethnicity for the sample was recorded as: White British $(N=133)$, White English $(N=6)$, Irish $(N=11)$, White Other $(N=20)$, Black Caribbean $(N=19)$, Black British $(N=48)$, Black African $(N=37)$, Other African $(N=12)$, Indian $(N=6)$, Pakistani $(N=1)$, Bangladeshi $(N=3)$, Other $(N=57)$, and with 33 patients recorded as having missing data for ethnicity.

Mean PSYRATS-AH total score was 27.57 ( $S D=6.42$; range 2-43). Primary diagnoses were only recorded in the research database of the clinic from 2013 meaning diagnoses are not available for all patients. From available data, primary diagnoses were recorded as Schizophrenia $(N=84)$, Depressive/mood disorder $(N=27)$, anxiety disorder $(N=10)$, bipolar disorder $(N=6)$, PTSD $(N=5)$ and other neurotic disorder $(N=1)$. Secondary and subsequent diagnoses were not available. Antipsychotic prescribing was not recorded in the research database, however, a previously conducted clinical trial of psychological therapy with participants referred to the same service reported an antipsychotic medication prevalence of $96 \%$ [39].

\section{Network structure}

The network is displayed in Fig. 1. All nodes form part of the network except the item 'belief regarding the origin of voices' (BEL) which is isolated due to having estimated edges of value zero with other nodes. Although Location (LOC) does form part of the overall network it is connected by a single edge of low edge weight and has an $R^{2}$ predictability metric of 0 . These items score lowest for

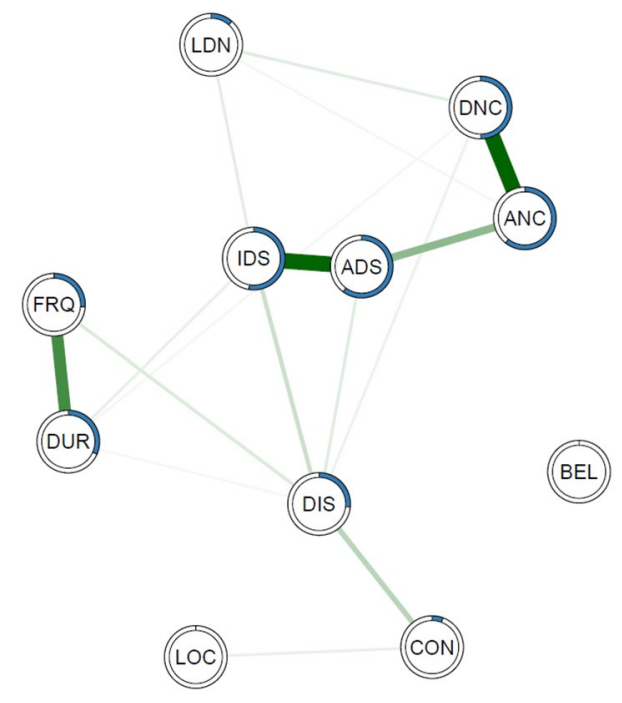

Fig. 1 MGM predictability networks of PSYRATS-AH items of auditory hallucinations. The thickness of the line indicates the strength of the relationship between nodes. Blue ring around each node represents the proportion of the variance of this item explained by all other items in the network. PSYRATS-AH items: CON controllability of voices, $L O C$ location, $B E L$ belief re: origin of voices, $D U R$ duration, $D I S$ disruption to life caused by voices, $L D N$ loudness, $F R Q$ frequency, IDS intensity of distress, $A D S$ amount of distress, $A N C$ amount of negative content, $D N C$ degree of negative content

overall expected influence $(\mathrm{BEL}=-1.45, \mathrm{LOC}=-1.29)$. The additional appraisal item Controllability (CON) has a relatively low expected influence $(-0.81 ; 8$ th out of 11 nodes) and predictability ( $R^{2}$ predictability of $\left.5.7 \%\right)$ although it does contribute to overall network structure. Notably, it is most strongly connected to disruption to life (DIS) suggesting a mutual interaction between these nodes. Numeric predictability scores are reported in Table S1 of the supplementary material (Fig. 2).

The most central, influential, and most highly predicted by variation in other network nodes are typically those nodes related to distress. Intensity of distress (IDS), amount of distress (ADS) and amount of negative content (ANC) are estimated to be the most influential nodes in the network as well as those with values that are most predicted by the values of the other nodes in the network. Notably, disruption of life (DIS) has the second highest value in terms of closeness (1.07) and betweenness (1.10), following IDS, which indicates it has the second largest average distance from all other nodes in the network.

\section{Reliability of network estimates}

The results of bootstrapped values for edge-weight reliability are included in the Supplementary Materials. 
Fig. 2 Centrality metrics for PSYRATS-AH items in network of auditory hallucinations, ordered by Expected Influence
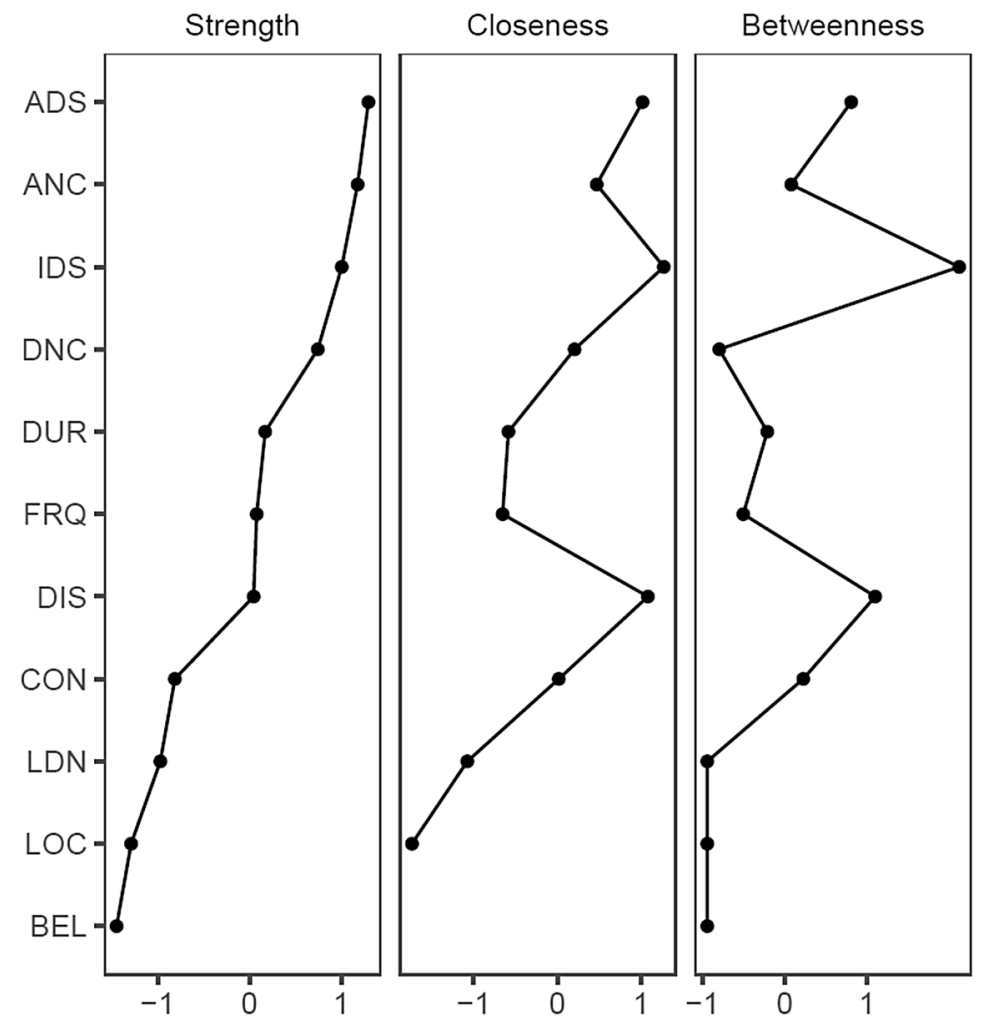

Expectedlnfluence

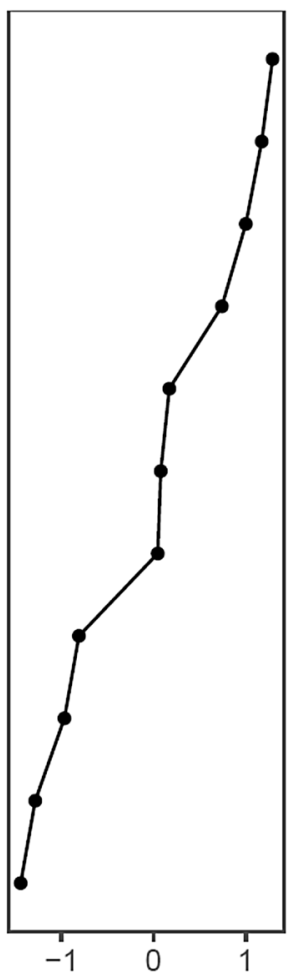

\section{Study 2: Temporal stability of network structure for hallucinated voices}

\section{Descriptive statistics}

Of the 204 included patients, 104 (51.2\%) were male, 99 $(48.8 \%)$ were female and one person had missing data. The mean age of patients was $40.95(\mathrm{SD}=9.74)$. Ethnicity for the sample was recorded as: White British $(N=67)$, White English $(N=5)$, Irish $(N=7)$, White Other $(N=11)$, Black Caribbean $(N=10)$, Black British $(N=30)$, Black African $(N=21)$, Other African $(N=7)$, Indian $(N=3)$, Pakistani $(N=1)$, Bangladeshi $(N=3)$, Other $(N=31)$ with eight patients recorded as missing data.

As previously, diagnoses are only available for a subset of patients. Primary diagnoses for this sample was recorded as: Schizophrenia $(N=39)$, depressive/mood disorder $(N=20)$, anxiety disorder $(N=6)$, bipolar disorder $(N=5)$, PTSD $(\mathrm{N}=1)$ and other neurotic disorder $(N=1)$.

The mean time period between the two assessments was 117.2 days ( $\mathrm{SD}=68.4$; range $23-524)$.

An independent samples $t$ test indicated that the patients who did not have data for a second assessment and so were excluded from Study 2, were not significantly different in their total PSYRATS-AH scores from patients who were included ( $t=0.397, p=0.691$, Cohen's $d=0.041)$.

\section{Change in scale scores}

Mean total PSYRATS-AH score at assessment one was 27.69 $(\mathrm{SD}=5.92)$, at assessment two it was $25.92(\mathrm{SD}=8.36)$. Total PSYRATS-AH scores from the first and second assessment correlated $(r=0.59, p<0.001)$ although when tested with a paired samples $t$ test the difference between scores was significant $(t=3.72 ; p=0.0003)$ with an effect size calculated with a Cohen's $d$ of 0.24 indicating a statistically significant decline of small effect between the two assessments.

\section{Network structure}

Networks with $R^{2}$ predictability for each PSYRATS-AH item at assessments one and two are displayed in Fig. 3. The $R^{2}$ predictability values ordered by extent of difference are displayed in Table 1.

\section{Centrality metrics}

Centrality metrics for both networks are displayed in Fig. 4. Centrality metrics tended to be similar across the two assessments, almost identical for strength and estimated influence estimates. Betweenness centrality was most dissimilar between time points although this metric is most 


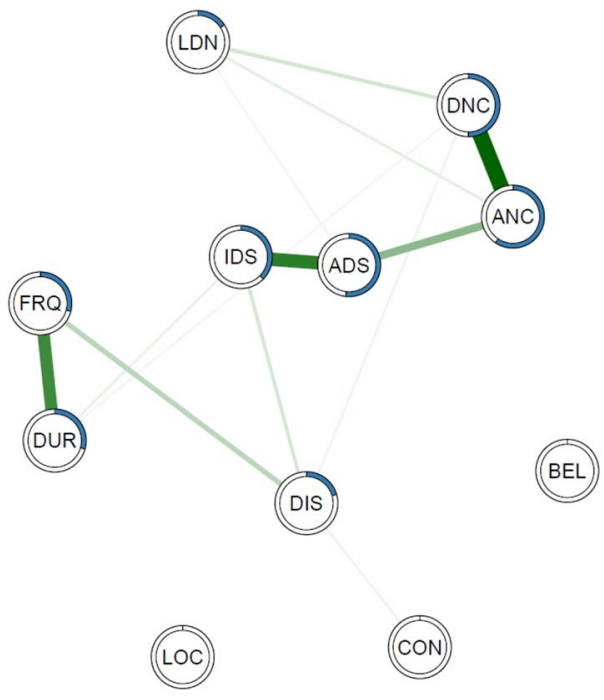

(a) First assessment

Fig. 3 Estimate networks of PSYRATS-AH items on a first and b second assessment of auditory hallucinations over a period of nonintervention. The thickness of the line indicates the strength of the relationship between nodes. Blue ring around each node represents the proportion of the variance of this item explained by all other

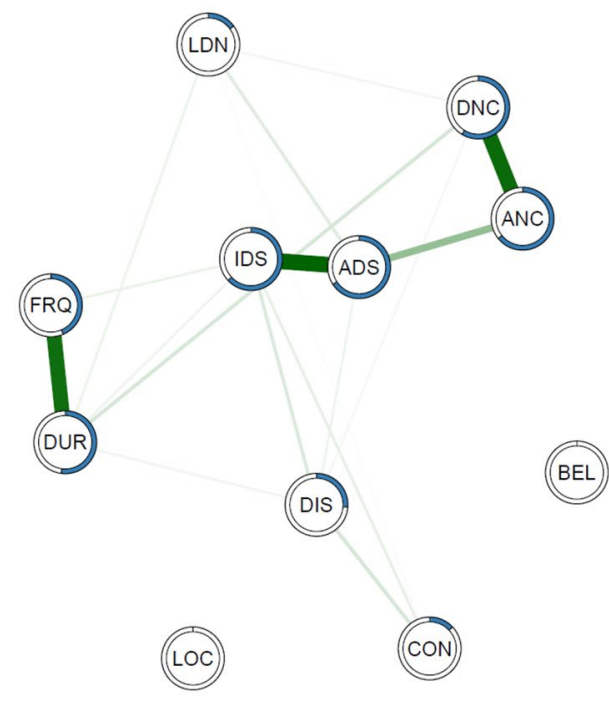

(b) Second assessment

items in the network. PSYRATS-AH items: $C O N$ controllability of voices, $L O C$ location, $B E L$ belief about the origin of voices, $D U R$ duration, $D I S$ disruption to life caused by voices, $L D N$ loudness, $F R Q$ frequency, IDS intensity of distress, $A D S$ amount of distress, ANC amount of negative content, $D N C$ degree of negative content
Table $1 R^{2}$ predictability for items at assessments one and two ordered by difference

\begin{tabular}{llll}
\hline psyrats-ah item & $\begin{array}{l}\text { 1st assessment } \\
R^{2} \text { predictability } \\
(\%)\end{array}$ & $\begin{array}{l}\text { 2nd assessment } \\
R^{2} \text { predictability } \\
(\%)\end{array}$ & Difference (\%) \\
\hline Intensity of distress (IDS) & 37.4 & 63.1 & 25.7 \\
Duration (DUR) & 29.4 & 52.1 & 22.7 \\
Frequency (FRQ) & 29.1 & 43.6 & 14.5 \\
Amount of distress (ADS) & 51.8 & 65.8 & 14.0 \\
Controllability (CON) & 0.0 & 13.0 & 13.0 \\
Degree of negative content (DNC) & 50.1 & 58.7 & 8.6 \\
Disruption to life (DIS) & 20.5 & 26.2 & 5.7 \\
Amount of negative content (ANC) & 59.4 & 64.2 & 4.8 \\
Loudness (LDN) & 15.8 & 15.0 & 0.8 \\
Belief about the origin of voices (BEL) & 0.0 & 0.0 & 0.0 \\
Location (LOC) & 0.0 & 0.0 & 0.0
\end{tabular}

sensitive to minor changes in network structure [40]. Loudness (LDN) and 'Belief about origin' (BEL) have incomplete closeness metrics due to being isolated nodes in the networks.

\section{Differences in global connectivity over time}

The results of the NetworkComparisonTest between assessments one and two showed the maximum difference between any edge weight was $0.27(p=0.045)$. However, we note the $\mathrm{p}$ value is only marginally below the traditional cut-off of $p<0.05$. Post-hoc tests showed 7 out of 121 edges $(5.77 \%)$ had significantly different weights between the two networks with uncorrected $p$ values of less than 0.05 . These were: belief about the origin of voices-controllability, belief about the origin of voices-location, belief about the origin of voices-disruption to life, belief about the origin of voices - frequency, frequency — duration, frequencyintensity of distress, intensity of distress - controllability. However, none survived Holm-Bonferroni correction for multiple comparisons. 
Fig. 4 Centrality metrics estimated for auditory hallucinations networks at assessment one and assessment two. Centrality indices are shown as standardized $Z$ scores to facilitate comparisons between different networks

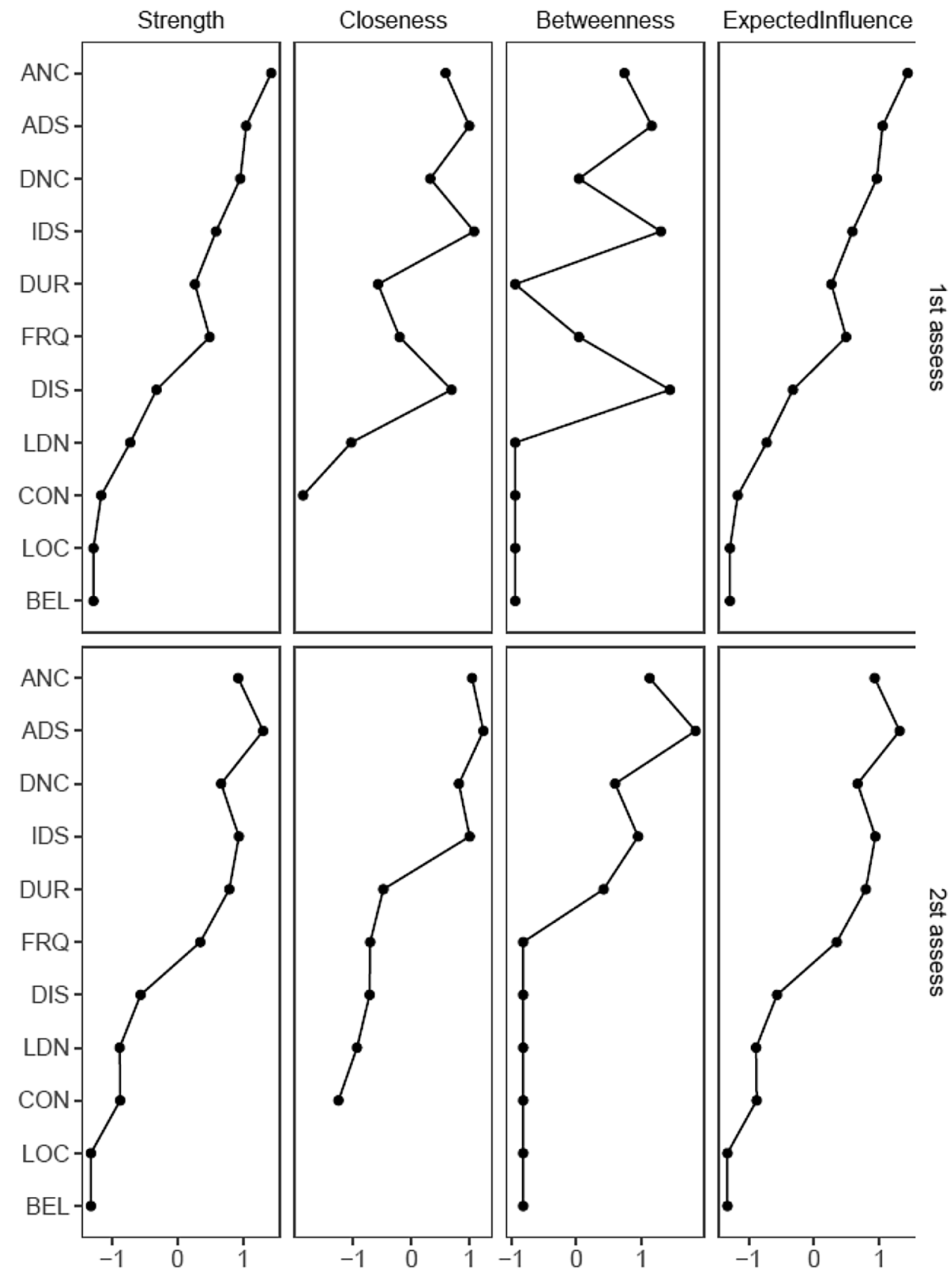

The two networks showed a difference in global strength values with a global strength value of 2.95 for the first assessment network and 5.10 for the second assessment network ( $p=0.0025)$, indicating that the overall connectedness of the second assessment network is increased by 2.15 points compared to the first assessment network. Given the marginal significance of the results of the network structure comparison and lack of difference for specific edge weight difference after correction we conclude conservatively that it is unlikely we can confidently reject the null hypothesis that the networks do not differ. The change in global strength is more convincing and likely reflects the change in PSYRATS-AH total score.

\section{Reliability of network estimates}

The results of bootstrapped values for edge-weight reliability for both graphs are included in the Supplementary Materials. Given the smaller sample size, as expected, the edge-weight reliability estimates for Study 2 show wider ranges and therefore likely lower reliability than those reported in Study 1. 


\section{Discussion}

In this study, insight and controllability appraisal items made relatively small contributions to the overall network structure and to the most clinically significant components with the most central and predictable components tending to reflect distress. The only component that was less influential and predictable than these items was the perceived location of the voice (inside or outside the head), which seemed to be irrelevant to the overall network. In terms of temporal stability, the overall severity score comparison showed a modest statistically significant decrease over the period between assessments and there was evidence of a small change in global strength. However, there was no convincing evidence for an alteration in structure and the appraisal items remained as the least influential.

The fact that insight-related beliefs measured by the PSYRATS-AH (perceived location and belief about origin) contributed almost nothing to the overall network structure either in terms of centrality of predictability raises additional questions about the clinical utility of these criteria in distinguishing 'clinically significant' or 'pathological' voices $[4,41,42]$. In terms of 'controllability' of voices, this item had a modest but detectable potential influence on the network and was most connected to disruption caused by the voices in everyday life. Existing studies on perceived controllability of voices suggests that it is higher in non-clinical voice hearers $[14,43]$ and in patients with pleasurable voices [44]. Nevertheless, it is not possible to conclude with confidence from our data whether the relationship reflects reverse causality from disruption rather than a measure of metacognitive control. One potential indicator may be that controllability remained low in centrality across the two time points but increased in predictability, potentially suggesting that it is more likely to be affected by, rather than affecting, the network structure of hallucinated voices.

We also note the consistently high centrality and predictability of items relating to distress and negative content. It is also worth noting here that distress-related items remained high in centrality across the two time periods in the longitudinal study, but in terms of predictability, the Intensity of Distress item showed the most change over time out of all the items with other distress and negative content items showing more modest changeability. This may suggest that reducing distress and negative content might be effective in reducing the network of hallucinated voice components as a whole, although given the limited evidence for potential role of controllability and belief about origin, which are common targets for psychological therapy aimed at reducing distress [45], alternative targets may be needed. Considering that the items measuring the frequency and duration of voices seem to show the highest levels of predictability and centrality after the distress items, these may be suitable candidates, although the extent to which they drive distress rather than reflect distress-causing processes remain unclear [46].

Importantly, despite the small to absent contribution of appraisals to the overall experience of hallucinated voices, this does not discount the possibility that other appraisals, evaluations or metacognitive beliefs and processes (reality monitoring, executive control and so on) still play a more central role. For instance, the PSYRATS-AH does not include key appraisals about voices such as omnipotence, identity and social-rank beliefs that are hypothesised to be central to the experience of hearing voices and drive distress and disability [47]. Indeed, a cognitive behavioural therapy intervention focusing on beliefs regarding the 'power' and 'malevolence' of voices has been successful in reducing compliance with command hallucinations $[48,49]$ and it may be that these or other appraisals relating to other factors in hallucinated voices-like depth of agency or the extent to which voices seem to have individuated and characterful identities [50-52], rather than the ones measured here, are more relevant for driving distress more generally.

We also note in the longitudinal analysis that the overall severity of hallucinations reduced modestly with no convincing evidence for differences in network structure. This study did not evaluate a period of treatment change in the clinic in which it was conducted, and given that a prior study conducted in the same clinic found most patients are on stable doses of antipsychotic medication [39], we suspect that this change may reflect non-treatment-related fluctuation in the experience of auditory hallucinations-particularly given that wait lists, at least in randomised controlled trials, have been found to be modestly counter-therapeutic [53], contrary to what we found here. Previous studies have tended to show marked fluctuation over short time periods (typically over several days; [54-57]) with more stability when measured over a period of a year or more $[58,59]$ and it is possible that the results presented here covering an average time period of 117.2 days are the mid-point in this pattern of temporal fluctuation. However, we also note the wide range of time periods between assessments that were summarised by this mean value and more precise, time-stratified studies are needed to test this assumption more reliably. We also raise the question of whether a more radical change in structure would be apparent if networks were compared before and after treatment. Network structure has been found to predict treatment outcome in depression [36] and it is possible that this may be the case for hallucinated voices.

It is worth noting some potential limitations to the study reported here. The data are taken from routine clinical practice and may not have the same level of reliability as a specifically designed prospective study. In the longitudinal 
analysis, dropouts may also affect the final population of patients whose data are included in the study. Indeed, as the data were drawn from an outpatient service, this naturally selects for referrals of people who are less likely to be experiencing an acute episode of psychosis and who live in the community, which may mean the results are less representative of voices in acute episodes. Conversely, those whose difficulties remit or who decide the treatment offered by the clinic does not suit them may also be less likely to return. Although previous data shows most patients from the clinic from which the sample was drawn are on stable doses of antipsychotic medication [39], the lack of data on prescribing meant we could not estimate to what extent this affected symptom variability. Although not a limitation per se, we note that the transdiagnostic nature of this study does provide data on potential differences in the structure of hallucinated voices that are specific to certain diagnoses.

\section{Conclusions}

We report that several appraisals and beliefs that have previously been identified as key in characterising the clinical significance of hallucinated voices in psychiatry seem to have little relation to several measures of distress and disability and seem to make little contribution to the estimated network structure. The structure of hallucinated voices changed little over time.

Acknowledgements The authors would like to thank Ciarán O'Driscoll for his advice when conducting this study.

Author contributions EP (1st author) and VB conceived the study and analysed and interpreted the data. EP (1st author) drafted the manuscript. All authors read and approved the final manuscript.

Funding None reported.

\section{Compliance with ethical standards}

Conflict of interest The authors declare that they have no competing interests.

Ethics approval and consent to participate Date was collected in routine clinical practice. Ethical approval for the use of these data in research was granted by the London-Dulwich Research Ethics Committee (ref: 15/LO/1831) and only patients who consent to their anonymised data being used in research are included in the database.

Consent for publication No personal data of an individual is included in this study.

Availability of data and materials The datasets generated and/or analysed during the current study are available in the Open Science Framework repository https://osf.io/qkn6z/
Open Access This article is licensed under a Creative Commons Attribution 4.0 International License, which permits use, sharing, adaptation, distribution and reproduction in any medium or format, as long as you give appropriate credit to the original author(s) and the source, provide a link to the Creative Commons licence, and indicate if changes were made. The images or other third party material in this article are included in the article's Creative Commons licence, unless indicated otherwise in a credit line to the material. If material is not included in the article's Creative Commons licence and your intended use is not permitted by statutory regulation or exceeds the permitted use, you will need to obtain permission directly from the copyright holder. To view a copy of this licence, visit http://creativecommons.org/licenses/by/4.0/.

\section{References}

1. Upthegrove R, Broome MR, Caldwell K, Ives J, Oyebode F, Wood SJ (2015) Understanding auditory verbal hallucinations: a systematic review of current evidence. Acta Psychiatr Scand

2. Larøi F, Sommer IE, Blom JD, Fernyhough C, Ffytche DH, Hugdahl K et al (2012) The characteristic features of auditory verbal hallucinations in clinical and nonclinical groups: state-of-the-art overview and future directions. Schizophr Bull 38:724-733

3. Berrios GE, Dening TR (1996) Pseudohallucinations: a conceptual history. Psychol Med 26:753-763

4. Copolov D, Trauer T, Mackinnon A (2004) On the non-significance of internal versus external auditory hallucinations. Schizophr Res 69:1-6

5. Garety PA, Kuipers E, Fowler D, Freeman D, Bebbington PE (2001) A cognitive model of the positive symptoms of psychosis. Psychol Med 31:189-195

6. Stephane M, Kuskowski M, McClannahan K, Surerus C, Nelson K (2010) Evaluation of inner-outer space distinction and verbal hallucinations in schizophrenia. Cognit Neuropsychiatry $15: 441-450$

7. Docherty NM, Dinzeo TJ, McCleery A, Bell EK, Shakeel MK, Moe A (2015) Internal versus external auditory hallucinations in schizophrenia: symptom and course correlates. Cognit Neuropsychiatry 20:187-197

8. Oulis PG, Mavreas VG, Mamounas JM, Stefanis CN (1995) Clinical characteristics of auditory hallucinations. Acta Psychiatr Scand 92:97-102

9. Nayani TH, David AS (1996) The auditory hallucination: a phenomenological survey. Psychol Med 26:177-189

10. Oyebode F (2008) Sims' symptoms in the mind: an introduction to descriptive psychopathology. Elsevier Health Sciences, Amsterdam

11. Taylor MA, Vaidya NA (2008) Descriptive psychopathology: the signs and symptoms of behavioral disorders. Cambridge University Press, Cambridge

12. Morrison AP, Haddock G, Tarrier N (1995) Intrusive thoughts and auditory hallucinations: a cognitive approach. Behav Cognit Psychother 23:265-280

13. Varese F, Bentall RP (2011) The metacognitive beliefs account of hallucinatory experiences: a literature review and meta-analysis. Clin Psychol Rev 31:850-864

14. Baumeister D, Sedgwick O, Howes O, Peters E (2017) Auditory verbal hallucinations and continuum models of psychosis: a systematic review of the healthy voice-hearer literature. Clin Psychol Rev 51:125-141

15. Borsboom D, Cramer AOJ (2013) Network analysis: an integrative approach to the structure of psychopathology. Annu Rev Clin Psychol 9:91-121 
16. Fried EI, van Borkulo CD, Cramer AOJ, Boschloo L, Schoevers RA, Borsboom D (2017) Mental disorders as networks of problems: a review of recent insights. Soc Psychiatry Psychiatr Epidemiol 52:1-10

17. Isvoranu A-M, Borkulo CDV, Boyette L-L, Wigman JTW, Vinkers $\mathrm{CH}$ et al (2017) A Network approach to psychosis: pathways between childhood trauma and psychotic symptoms. Schizophr Bull. 43:187-196

18. Bell V, O'Driscoll C (2018) The network structure of paranoia in the general population. Soc Psychiatry Psychiatr Epidemiol 53:737-744

19. Murphy J, McBride O, Fried E, Shevlin M (2018) Distress, impairment and the extended psychosis phenotype: a network analysis of psychotic experiences in an US general population sample. Schizophr Bull 44:768-777

20. van Rooijen G, Isvoranu A-M, Meijer CJ, van Borkulo CD, Ruhé HG, de Haan L (2017) A symptom network structure of the psychosis spectrum. Schizophr Res 189:75-83

21. Haddock G, McCARRON J, Tarrier N, Faragher EB (1999) Scales to measure dimensions of hallucinations and delusions: the psychotic symptom rating scales (PSYRATS). Psychol Med 29:879-889

22. Santos HP, Kossakowski JJ, Schwartz TA, Beeber L, Fried EI (2018) Longitudinal network structure of depression symptoms and self-efficacy in low-income mothers. PLoS ONE 13:e0191675

23. Peters E, Crombie T, Agbedjro D, Johns LC, Stahl D, Greenwood $\mathrm{K}$ et al (2015) The long-term effectiveness of cognitive behavior therapy for psychosis within a routine psychological therapies service. Front Psychol. https://doi.org/10.3389/fpsyg.2015.01658

24. Woodward TS, Jung K, Hwang H, Yin J, Taylor L, Menon M et al (2014) Symptom dimensions of the psychotic symptom rating scales in psychosis: a multisite study. Schizophr Bull 40(Suppl_4):S265-S274

25. Haslbeck JM, Waldorp LJ (2015) mgm: Structure estimation for time-varying mixed graphical models in high-dimensional data. J Stat Softw

26. Tibshirani R (1996) Regression shrinkage and selection via the Lasso. J R Stat Soc Ser B (Methodol) 58:267-288

27. Friedman J, Hastie T, Tibshirani R (2010) Regularization paths for generalized linear models via coordinate descent. J Stat Softw 33:1-22

28. Haslbeck JMB, Fried EI (2017) How predictable are symptoms in psychopathological networks? A reanalysis of 18 published datasets. Psychol Med 47:2767-2776

29. Haslbeck JMB, Waldorp LJ (2018) How well do network models predict observations? On the importance of predictability in network models. Behav Res 50:853-861

30. Epskamp S, Cramer AO, Waldorp LJ, Schmittmann VD, Borsboom D (2012) qgraph: network visualizations of relationships in psychometric data. J Stat Softw 48:1-18

31. Fruchterman TMJ, Reingold EM (1991) Graph drawing by forcedirected placement. Softw Pract Exp 21:1129-1164

32. Epskamp S, Borsboom D, Fried EI. Estimating psychological networks and their accuracy: a tutorial paper. 2016. arxiv: 16040 8462 [stat]. Accessed 25 Sep 2016

33. Robinaugh DJ, Millner AJ, McNally RJ (2016) Identifying highly influential nodes in the complicated grief network. J Abnorm Psychol 125:747-757

34. van Borkulo C, Boschloo L, Kossakowski JJ, Tio P, Schoevers RA, Borsboom D (2017) Comparing network structures on three aspects: a permutation test. 26. Available from: https://www.resea rchgate.net/publication/314750838. Cited

35. van Borkulo C (2019) cvborkulo/NetworkComparisonTest. R. https://github.com/cvborkulo/NetworkComparisonTest. Accessed 16 Dec 2019
36. van Borkulo C, Boschloo L, Borsboom D, Penninx BWJH, Waldorp LJ, Schoevers RA (2015) Association of symptom network structure with the course of depression. JAMA Psychiatry 72:1219-1226

37. Epskamp S, Fried EI (2015) bootnet: Bootstrap methods for various network estimation routines. R-Package. Available at: https:// rdrr.io/cran/bootnet

38. Fried EI, von Stockert S, Haslbeck JMB, Lamers F, Schoevers RA, Penninx BWJH (2019) Using network analysis to examine links between individual depressive symptoms, inflammatory markers, and covariates. Psychol Med 1-9

39. Peters E, Landau S, McCrone P, Cooke M, Fisher P, Steel C et al (2010) A randomised controlled trial of cognitive behaviour therapy for psychosis in a routine clinical service. Acta Psychiatr Scand 122:302-318

40. Epskamp S, Borsboom D, Fried EI (2018) Estimating psychological networks and their accuracy: a tutorial paper. Behav Res 50:195-212

41. Connors MH, Robidoux S, Langdon R, Coltheart M (2016) Beliefs about hearing voices. Conscious Cogn 43:89-101

42. Peters E, Ward T, Jackson M, Woodruff P, Morgan C, McGuire $P$ et al (2017) Clinical relevance of appraisals of persistent psychotic experiences in people with and without a need for care: an experimental study. Lancet Psychiatry 4:927-936

43. Moritz S, Larøi F (2008) Differences and similarities in the sensory and cognitive signatures of voice-hearing, intrusions and thoughts. Schizophr Res 102:96-107

44. Sanjuan J, Gonzalez JC, Aguilar EJ, Leal C, van Os J (2004) Pleasurable auditory hallucinations. Acta Psychiatr Scand 110:273-278

45. Thomas N, Hayward M, Peters E, van der Gaag M, Bentall RP, Jenner J et al (2014) Psychological therapies for auditory hallucinations (voices): current status and key directions for future research. Schizophr Bull 40(Suppl_4):S202-S212

46. Larøi F, Thomas N, Aleman A, Fernyhough C, Wilkinson S, Deamer $F$ et al (2019) The ice in voices: understanding negative content in auditory-verbal hallucinations. Clin Psychol Rev 67:1-10

47. Chadwick P, Birchwood M (1994) The omnipotence of voices: a cognitive approach to auditory hallucinations. Br J Psychiatry 164:190-201

48. Birchwood M, Michail M, Meaden A, Tarrier N, Lewis S, Wykes T et al (2014) Cognitive behaviour therapy to prevent harmful compliance with command hallucinations (COMMAND): a randomised controlled trial. Lancet Psychiatry 1:23-33

49. Birchwood M, Dunn G, Meaden A, Tarrier N, Lewis S, Wykes T et al (2018) The COMMAND trial of cognitive therapy to prevent harmful compliance with command hallucinations: predictors of outcome and mediators of change. Psychol Med 48:1966-1974

50. Bell V (2013) A community of one: social cognition and auditory verbal hallucinations. PLoS Biol 11:e1001723

51. Wilkinson S, Bell V (2016) The representation of agents in auditory verbal hallucinations. Mind Lang 31:104-126

52. Bell V, Mills KL, Modinos G, Wilkinson S (2017) Rethinking social cognition in light of psychosis: reciprocal implications for cognition and psychopathology. Clin Psychol Sci 5:537-550

53. Patterson B, Boyle MH, Kivlenieks M, Van Ameringen M (2016) The use of waitlists as control conditions in anxiety disorders research. J Psychiatr Res 83:112-120

54. Delespaul P, deVries M, van Os J (2002) Determinants of occurrence and recovery from hallucinations in daily life. Soc Psychiatry Psychiatr Epidemiol 37:97-104

55. Kimhy D, Delespaul P, Corcoran C, Ahn H, Yale S, Malaspina D (2006) Computerized experience sampling method (ESMc): assessing feasibility and validity among individuals with schizophrenia. J Psychiatr Res 40:221-230 
56. Kimhy D, Wall MM, Hansen MC, Vakhrusheva J, Choi CJ, Delespaul P et al (2017) Autonomic regulation and auditory hallucinations in individuals with schizophrenia: an experience sampling study. Schizophr Bull 43:754-763

57. Schlier B, Hennig T, Lincoln TM (2017) Measuring fluctuations across the Continuum of Auditory Hallucinations. Development and validation of a state inventory. Psychiatry Res 253:325-332
58. Csipke E, Kinderman P (2006) A longitudinal investigation of beliefs about voices. Behav Cognit Psychother 34:365-369

59. Hartigan N, McCarthy-Jones S, Hayward M (2014) Hear today, not gone tomorrow? An exploratory longitudinal study of auditory verbal hallucinations (hearing voices). Behav Cognit Psychother $42: 117-123$ 